\title{
Measurement and simulation of the polarization-dependent Purcell factor in a microwave fishnet metamaterial
}

\author{
Kaizad Rustomji, ${ }^{1,2,{ }^{*}}$ Redha Abdeddaim, ${ }^{1}$ C. Martijn de Sterke, ${ }^{2}$ Boris Kuhlmey, ${ }^{2}$ and Stefan Enoch ${ }^{1}$ \\ ${ }^{1}$ Aix Marseille Univ, CNRS, Centrale Marseille, Institut Fresnel, \\ UMR 7249, 13013 Marseille, France \\ ${ }^{2}$ Centre for Ultrahigh Bandwidth Devices for Optical Systems and Institute of Photonics and Optical Science, \\ School of Physics, University of Sydney, Sydney, NSW 2006, Australia
}

(Received 6 October 2016; revised manuscript received 15 December 2016; published 31 January 2017)

\begin{abstract}
We determine, experimentally and numerically, the electric and magnetic Purcell factors in a fishnet metamaterial in the frequency range $5-15 \mathrm{GHz}$ by measuring the impedance of a dipole antenna. We compare measurements and numerical simulations of the Purcell factor for transverse electric $\left(\mathrm{TE}_{\mathrm{z}}\right)$ and transverse magnetic $\left(\mathrm{TM}_{\mathrm{z}}\right)$ polarizations. For $\mathrm{TM}_{\mathrm{z}}$ polarization, the dispersion relation of the structure is hyperbolic and enhances the Purcell factor. For $\mathrm{TE}_{\mathrm{z}}$ polarization, the dispersion relation does not allow any propagating solutions and decreases the Purcell factor below the effective plasma frequency. Eigenmode calculations of the periodic unit cell of the metamaterial are used to obtain the band structure and confirm the presence of hyperbolic isofrequency surfaces. The isofrequency surfaces are used to calculate the density of states (DOS). We also use the impedance method to obtain the DOS by averaging the Purcell factor obtained at different locations over the periodic unit cell and find good agreement with DOS calculated from eigenmode calculations.
\end{abstract}

DOI: 10.1103/PhysRevB.95.035156

\section{INTRODUCTION}

In the paper of Purcell [1] it was shown that the spontaneous emission rate of an emitter is modified by its environment. The ratio of the rate of spontaneous emission in a specific environment to that in free space is called the Purcell factor $(F)$. Modification of the rate of spontaneous emission close to a conducting surface was observed experimentally in Refs. [2,3], by measuring the change in lifetime of fluorescent molecules near an interface. Inhibition of the rate of spontaneous emission in a waveguide was proposed first in Ref. [4] and observed experimentally in Rydberg atoms by Hulet et al. [5]. Inhibition of spontaneous emission by placing emitters in the band gap of photonic crystals was proposed by Yablonovitch [6]. The control of the rate of spontaneous emission was achieved experimentally in photonic crystals in Refs. [7-9]. Engineering the Purcell factor is of interest in developing single-photon emitters [10-12] and lasers [13]. Controlling the rate of spontaneous emission has been studied in nanoresonators [14-17], microcavities [18], and more recently in hyperbolic metamaterials (HMM) $[19,20]$. Hyperbolic metamaterials have gained attention because of the possibility of achieving a large density of states (DOS) due to a hyperbolic dispersion in the TM polarization. A property of hyperbolic media which has received little attention is the polarization dependence of the Purcell factor: while there is an enhancement in the Purcell factor for TM polarization, for TE polarization no propagating modes are permitted and the Purcell factor is suppressed. We report experimental confirmation of this property and provide numerical calculations of the same. Thus far, Purcell factor measurements in the microwave for HMMs have been carried out for a wire medium $[21,22]$ which is characterized in literature as a type I HMM [20] metamaterial; we focus on type II HMM composed of conducting sheets.

\footnotetext{
*kaizad.rustomji@fresnel.fr
}

The Purcell factor is usually studied by measuring the change in decay lifetimes of quantum dots or fluorescent emitters. It was shown in Ref. [23] that the impedance of an antenna can also be used to obtain the Purcell factor. Since antennas can be used for microwave frequencies, it is possible to measure the Purcell factor for large structures with this method. The impedance method was applied to measure the Purcell factor close to a conducting plate [24] at microwave frequencies and was found in agreement with theoretical calculations. The impedance approach has also been applied numerically to study the Purcell factor for hyperbolic wire array metamaterials [22]. By replacing the electric dipole antenna with a magnetic dipole antenna, the magnetic Purcell factor can also be measured [25] directly with this method.

In this paper we experimentally measure the Purcell factor using the impedance of dipole antennas for a fishnet metamaterial, previously studied as an epsilon-near-zero material [26], in the frequency range 5-15 GHz. The uniaxial anisotropic structure has distinct dispersion relations for transverse electric $\left(\mathrm{TE}_{\mathrm{z}}\right)$ and transverse magnetic $\left(\mathrm{TM}_{\mathrm{z}}\right)$ polarizations, corresponding to ordinary and extraordinary modes. Further, we show that using magnetic and electric dipoles we can observe the effect of $\mathrm{TE}_{\mathrm{z}}$ and $\mathrm{TM}_{\mathrm{z}}$ dispersion relations on the Purcell factor. We use the impedance method to calculate the local and global DOS of our structure and compare it with the DOS calculated from the band structure.

This paper is structured as follows. In Sec. II we outline the theory of measurement of the Purcell factor from impedance of a classical dipole antenna. We then present our fishnet metamaterial structure and discuss its dispersion relations for $\mathrm{TM}_{\mathrm{z}}$ and $\mathrm{TE}_{\mathrm{z}}$ polarized fields in Sec. III. In Sec. IV we present the details of the numerical modeling using finite difference time domain (FDTD) software for simulations of the Purcell factor with finite-size dipole antenna in a finite metamaterial slab. Following that, eigenmode analysis of the unit cell of the infinite metamaterial is performed in Sec. V where the band structure is discussed and used to calculate DOS. Section VI 
presents the measurements of the Purcell factor using the impedance method for electric and magnetic dipoles in the metamaterial and comparison with FDTD simulations. We apply a method to filter out the reflections from the boundaries of the finite structure, which enables comparison with the infinite structure's DOS. In Sec. VII we compare the DOS obtained by the impedance method with the DOS obtained from eigenmode calculations.

\section{THEORY}

\section{A. Purcell effect}

The Purcell factor $(F)[1]$ is defined as $F=\gamma / \gamma_{0}$, where $\gamma$ is the rate of spontaneous emission in the medium and $\gamma_{0}$ is the rate of spontaneous emission in vacuum. If the optical dipole moment associated with transition is $\boldsymbol{\mu}$ then the spontaneous emission rate for an atom can be calculated using Fermi's golden rule [27]:

$$
\gamma=\frac{2 \omega}{3 \hbar \varepsilon_{0}}|\boldsymbol{\mu}|^{2} \rho\left(\mathbf{r}_{\mathbf{0}}, \omega\right)
$$

where the angular frequency is $\omega, \varepsilon_{0}$ is the permittivity of free space, $\hbar$ is the reduced Planck constant, and $\rho\left(r_{0}, \omega\right)$ is the local density of states (LDOS), which is proportional to the imaginary part of the Green's tensor at the location of the emitter $\mathbf{r}_{\mathbf{0}}$ :

$$
\rho\left(\mathbf{r}_{\mathbf{0}}, \omega\right)=\frac{6 \omega}{\pi c^{2}}\left[\mathbf{n}_{\mu} \cdot \operatorname{Im}\left\{\overleftrightarrow{\mathbf{G}}\left(\mathbf{r}_{\mathbf{0}}, \mathbf{r}_{\mathbf{0}} ; \omega\right)\right\} \cdot \mathbf{n}_{\mu}\right]
$$

Here $\mathbf{n}_{\mu}$ is the unit vector in the direction of the dipole moment and $c$ is the speed of light in vacuum. The Green's function can be of the electric or magnetic kind and depending upon the type can be expressed as a solution of the equations

$$
\nabla \times \nabla \times \overleftrightarrow{\mathbf{G}}^{e}\left(\mathbf{r}, \mathbf{r}_{\mathbf{0}} ; \omega\right)-\frac{\omega^{2}}{c^{2}}[\epsilon] \overleftrightarrow{\mathbf{G}}{ }^{e}\left(\mathbf{r}, \mathbf{r}_{\mathbf{0}} ; \omega\right)=\overleftrightarrow{\mathbf{I}} \delta\left(\mathbf{r}-\mathbf{r}_{\mathbf{0}}\right)
$$

for the electric Green's function $\overleftrightarrow{\mathbf{G}}^{e}\left(\mathbf{r}, \mathbf{r}_{\mathbf{0}} ; \omega\right)$ where $\overleftrightarrow{\mathbf{I}}$ is the unit dyad. Its magnetic counterpart is $\overleftrightarrow{\mathbf{G}}^{m}\left(\mathbf{r}, \mathbf{r}_{\mathbf{0}} ; \omega\right)$ given by the solution of

$$
\begin{aligned}
\nabla & \times[\varepsilon]^{-1} \nabla \times \overleftrightarrow{\mathbf{G}}^{m}\left(\mathbf{r}, \mathbf{r}_{\mathbf{0}} ; \omega\right)-\frac{\omega^{2}}{c^{2}} \overleftrightarrow{\mathbf{G}}^{m}\left(\mathbf{r}, \mathbf{r}_{\mathbf{0}} ; \omega\right) \\
& =\overleftrightarrow{\mathbf{I}} \delta\left(\mathbf{r}-\mathbf{r}_{\mathbf{0}}\right) .
\end{aligned}
$$

The Green's function allows the computation of electric field and magnetic fields from current densities by

$$
\begin{aligned}
& \mathbf{E}(\mathbf{r})=\mathbf{E}_{0}+\mathrm{i} \omega \mu \mu_{0} \int_{V} \overleftrightarrow{\mathbf{G}}^{e}\left(\mathbf{r}, \mathbf{r}^{\prime} ; \omega\right) \mathbf{j}_{e}\left(\mathbf{r}^{\prime}\right) d V^{\prime} \\
& \mathbf{H}(\mathbf{r})=\mathbf{H}_{0}+\mathrm{i} \omega \varepsilon \varepsilon_{0} \int_{V} \overleftrightarrow{\mathbf{G}}{ }^{m}\left(\mathbf{r}, \mathbf{r}^{\prime} ; \omega\right) \mathbf{j}_{m}\left(\mathbf{r}^{\prime}\right) d V^{\prime}
\end{aligned}
$$

where $\mathbf{j}_{e}\left(\mathbf{r}^{\prime}\right)$ and $\mathbf{j}_{m}\left(\mathbf{r}^{\prime}\right)$ denote the electric and magnetic current densities, respectively.

\section{B. Spontaneous emission and impedance of the classical dipole antenna}

The spontaneous emission rate obtained from Fermi's golden rule Eq. (1) can also be related to the power emitted by a classical ideal dipole [28]. The power emitted by a current source $\mathbf{j}$ is given by Ref. [29] as

$$
P=-\frac{1}{2} \int_{V} \operatorname{Re}\left\{\mathbf{j}^{*} \cdot \mathbf{E}\left(\mathbf{r}_{\mathbf{0}}\right)\right\} d V,
$$

for a time harmonic point dipole located at $\mathbf{r}_{\mathbf{0}}$ with a dipole moment $\boldsymbol{\mu}$, the current $\mathbf{j}=-\mathrm{i} \omega \boldsymbol{\mu} \delta\left(\mathbf{r}-\mathbf{r}_{\mathbf{0}}\right)$. The radiated power can then be expressed as $P=\frac{\omega}{2} \operatorname{Im}\left\{\boldsymbol{\mu}^{*} \cdot \mathbf{E}\left(\mathbf{r}_{\mathbf{0}}\right)\right\}$. Expressing the electric field at $\mathbf{r}_{\mathbf{0}}$ using the Green's function as $\mathbf{E}\left(\mathbf{r}_{\mathbf{0}}\right)=$ $\frac{\omega^{2}}{c^{2} \varepsilon_{0} \varepsilon} \overleftrightarrow{\mathbf{G}}\left(\mathbf{r}_{\mathbf{0}}, \mathbf{r}_{\mathbf{0}} ; \omega\right) \cdot \boldsymbol{\mu}$ we obtain the radiated power $\left(P_{\text {dip }}\right)$ as

$$
P_{\text {dip }}=\frac{\omega^{3}|\boldsymbol{\mu}|^{2}}{2 c^{2} \varepsilon_{0} \varepsilon}\left[\mathbf{n}_{\mu} \cdot \operatorname{Im}\{\overleftrightarrow{\mathbf{G}}\} \cdot \mathbf{n}_{\mu}\right]
$$

The spontaneous emission rate $\gamma$ from Eq. (1) and power emitted by a classical dipole Eq. (8) are thus related as $\gamma=$ $P_{\text {dip }} / \hbar \omega$. Hence the semiclassical analog of the Purcell factor, i.e., the modification of the rate of spontaneous emission, can be seen as the modification in power emitted by the dipole in the environment [30]:

$$
F=\gamma / \gamma_{0} \equiv P_{\text {dip }} / P_{\text {dip }, 0} .
$$

We point out that the quantum-mechanical dipole matrix element $\boldsymbol{\mu}^{q}=e\langle 2|r| 1\rangle$, where $e$ is the charge of the electron, $|2\rangle$ is the excited state, and $|1\rangle$ is the ground state, is linearly related to the classical dipole moment [31] $\boldsymbol{\mu}^{c}$ as $\boldsymbol{\mu}^{c}=2 \boldsymbol{\mu}^{q}$. However, we have used $\boldsymbol{\mu}$ to denote both as it does not affect Eq. (9). The power radiated by a weakly coupled subwavelength dipole antenna can be expressed using the current $(I)$ and the impedance of the dipole $(Z)$ :

$$
P_{\text {dip }}=\frac{1}{2}|I|^{2} \operatorname{Re}(Z) \text {. }
$$

The analogy stated by Eq. (9) along with Eq. (10) was used in Ref. [23] to show that, for a weakly coupled dipole antenna, the ratio of the radiated power near a structure to the radiated power in vacuum is equal to the ratio of the real component of the complex impedance $(Z)$, of the antenna near the structure and in vacuum. Furthermore, the $\operatorname{Re}(Z)$ can also be used to obtain the imaginary part of the Green's function and the LDOS. This allows us to obtain the Purcell factor $F$ through

$$
F^{e / m} \equiv \frac{\gamma}{\gamma_{0}}=\frac{\left[\mathbf{n}_{\mu} \cdot \operatorname{Im}\left\{\mathbf{G}^{e / m}\right\} \cdot \mathbf{n}_{\mu}\right]}{\left[\mathbf{n}_{\mu} \cdot \operatorname{Im}\left\{\mathbf{G}^{e / m}\right\} \cdot \mathbf{n}_{\mu}\right]_{0}}=\frac{\operatorname{Re}\left(Z^{e / m}\right)}{\operatorname{Re}\left(Z^{e / m}\right)_{0}} .
$$

Here $F^{e / m}$ is the electric and magnetic Purcell factors for an electric and magnetic dipole [see Eqs. (3) and (4)], respectively. It is apparent from Eq. (11) that measurements of the real part of an antenna's impedance can be used to obtain the Purcell factor.

\section{MICROWAVE FISHNET METAMATERIAL STRUCTURE}

We now present the fishnet metamaterial structure used in this paper. The structure is composed of copper grids in the $x-y$ plane stacked in the $\hat{z}$ direction (Fig. 1). The grids are separated in the $\hat{z}$ direction by expanded polystyrene which 


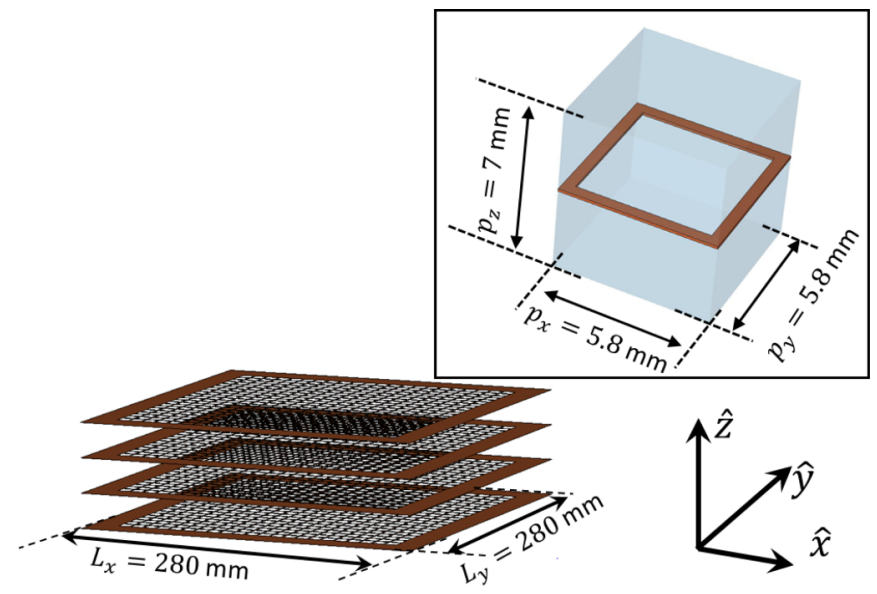

FIG. 1. The fishnet metamaterial structure used for study is composed of four layers of 0.1-mm-thick copper grids in the plane stacked in the $\hat{z}$ direction. Inset: Unit cell of the metamaterial structure.

has permittivity close to unity and negligible losses in the frequency range $5-15 \mathrm{GHz}$ used in this study. Copper has negligible losses in the frequency range in our study; this means that quenching, which is a consequence of ohmic losses in the metal, will not have a significant effect on the Purcell factor. The structure was fabricated using copper grids with the period in $x$ and $y$ directions $p_{x}=p_{y}=5.8 \mathrm{~mm}$ and spacing between the grids $p_{z}=7.0 \mathrm{~mm}$. The length of the inner edge is $4.95 \mathrm{~mm}$. The total length of the structure in the $x-y$ directions is $280 \mathrm{~mm}$. The frequency range for the measurements is 5-15 GHz, which corresponds to a wavelength range of 20-60 mm, which is consistent with the frequency range of our experimental apparatus (see Sec. VI).

The effective permittivity of this structure can be expressed by a uniaxial anisotropic permittivity tensor $[\bar{\varepsilon}]=\left[\varepsilon_{/ /}, \varepsilon_{/ /}, \varepsilon_{z z}\right]$ where the anisotropy axis of the structure is the $\hat{z}$ direction. In the simplest approximation, the effective permittivity of this structure can be described by a local, lossless Drude model [32-34] with $\varepsilon_{/ /}(\omega)=1-\omega_{p}^{2} / \omega^{2}$, where $\omega_{p}$ is the effective plasma frequency. For $\omega<\omega_{p}, \varepsilon_{/ /}<0$ whereas for $\omega>\omega_{p}$, $\varepsilon_{/ /}(\omega)>0$. The thickness of the copper sheets is $0.1 \mathrm{~mm}$, which is much smaller compared to the wavelength. Hence, the structure has an effective electromagnetic response of dilute thin metal layers in the $\hat{z}$ direction. This leads to the effective permittivity along $\hat{z}$ close to unity, and for the frequency range $5-15 \mathrm{GHz}, \varepsilon_{z z}(\omega) \simeq 1$.

This leads to an indefinite permittivity tensor with the property $\operatorname{Re}\left(\varepsilon_{/ /}\right) \cdot \operatorname{Re}\left(\epsilon_{z z}\right)<0$. For materials with such indefinite permittivity tensors the dispersion relation is dramatically different for $\mathrm{TE}_{\mathrm{z}}$ and $\mathrm{TM}_{\mathrm{z}}$ polarizations [35]. Our 5-15-GHz frequency range includes the effective plasma frequency which was reported around $14 \mathrm{GHz}$ for such a structure [26], so as to be able to observe the transition in the Purcell factor across the plasma frequency. We checked that the effective plasma frequency of $14 \mathrm{GHz}$ is consistent with that obtained from the Nicolson-Ross-Weir $[36,37]$ method of retrieval of effective electromagnetic parameters. At frequencies below $5 \mathrm{GHz}$ the wavelength becomes comparable to the size of the structure so finite-size effects become predominant, while above $15 \mathrm{GHz}$ the effective medium approximation starts to break down.

For a material with such a uniaxial anisotropic permittivity tensor, the propagating electromagnetic waves in the structure can be split into transverse electric $\left(\mathrm{TE}_{\mathrm{z}}\right)$ polarization for which the electric field $\mathbf{E}$ lies completely in the $x-y$ plane $\left(E_{z}=0\right)$ and transverse magnetic $\left(\mathrm{TM}_{\mathrm{z}}\right)$ polarization where the magnetic field $\mathbf{H}$ lies completely in the $x-y$ plane $\left(H_{z}=0\right)$. The isofrequency dispersion relations for the $\mathrm{TE}_{\mathrm{z}}$ and $\mathrm{TM}_{\mathrm{z}}$ polarizations are, respectively,

$$
\begin{aligned}
\mathrm{TE}: & \frac{k_{x}^{2}+k_{y}^{2}+k_{z}^{2}}{\varepsilon_{/ /}}=\frac{\omega^{2}}{c^{2}}, \\
\mathrm{TM}: & \frac{k_{x}^{2}+k_{y}^{2}}{\varepsilon_{z z}}-\frac{k_{z}^{2}}{\left|\varepsilon_{/ /}\right|}=\frac{\omega^{2}}{c^{2}} .
\end{aligned}
$$

The density of states is proportional to the infinitesimal volume between two closely separated isofrequency surfaces Eqs. (12) and (13) formed by frequencies $\omega$ and $\omega+\delta \omega$. If $\varepsilon_{/ /}(\omega)<0$ in the $\mathrm{TE}_{\mathrm{z}}$ polarization Eq. (12), there are no real propagating solutions, whereas $\mathrm{TM}_{\mathrm{z}}$ polarization Eq. (13) due to the hyperbolic nature of the dispersion relation can have a large Purcell factor [19,38-40]. Hence, the $\mathrm{TE}_{\mathrm{z}}$ and $\mathrm{TM}_{\mathrm{z}}$ polarizations have different Purcell factors. Since we shall study the Purcell factor using the impedance of dipoles, we now discuss the effect of polarization when the dipoles, electric and magnetic, are embedded inside such a uniaxial anisotropic structure.

In vacuum an electric dipole (oriented along $\hat{z}$ ) emits completely in the $\mathrm{TM}_{\mathrm{z}}\left(H_{z}=0\right)$ polarization; similarly a magnetic dipole (oriented along $\hat{z}$ ) emits in the $\mathrm{TE}_{\mathrm{z}}\left(E_{z}=0\right)$ polarization [41]. If the dipole is embedded in a general anisotropic medium, the fields cannot be decomposed simply into TE and TM polarizations. However, this decomposition becomes possible again in the particular case of a uniaxial medium where the dipole is oriented along the anisotropy axis $\hat{z}$. As shown by Clemmow [41], the electric and magnetic fields then retain the property $\left(H_{z}=0\right)$ and $\left(E_{z}=0\right)$ for electric and magnetic dipoles, respectively. Hence, for our particular anisotropic structure we can use electric and magnetic dipoles to observe the effect of dispersion relations in Eqs. (12) and (13) on the Purcell factor.

\section{NUMERICAL MODELLING FOR DOS CALCULATION}

For numerical calculations of the band structure and isofrequency dispersion relations, the periodic unit cell of the structure as shown in Fig. 1 was modeled and its propagating eigenmodes were calculated using the eigenmode solver available in commercial electromagnetic simulation software CST Microwave Studio [42]. Periodic boundary conditions are applied on the sides of the unit cell, which enforces that the structure is infinitely periodic in each direction. Individual phase shifts $\Delta \phi_{x}, \Delta \phi_{y}, \Delta \phi_{z}$ are applied on the boundaries, the phase shifts corresponding to phase difference between the fields on the two boundaries, and by varying the phase between zero and $\pi$ the propagation vector $\left(k_{x}, k_{y}, k_{z}\right)$ can be fixed to any location in the first Brillouin zone. The eigenfrequencies are then computed for the desired 


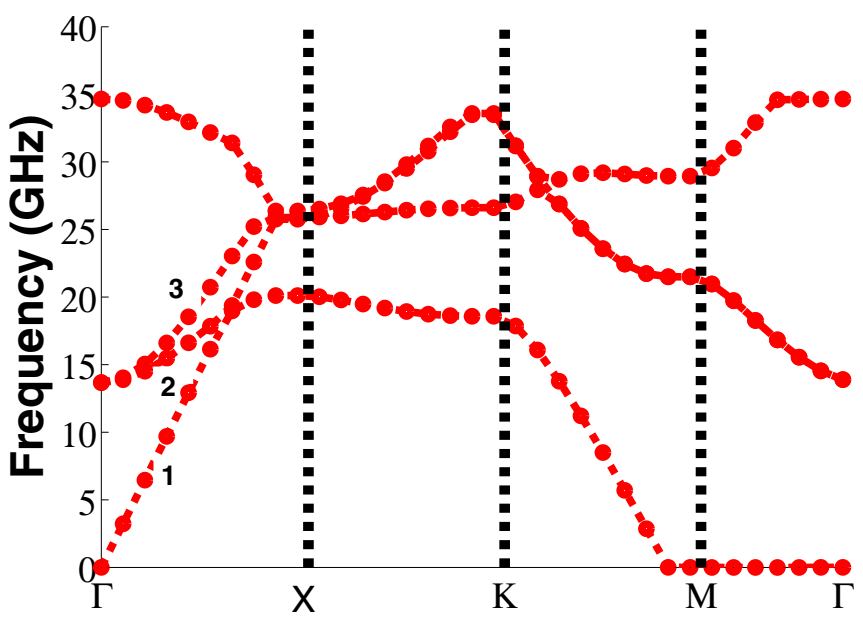

FIG. 2. Band diagram of the metamaterial unit cell in Fig. 1 along the path $\Gamma\left(k_{x}=0, k_{z}=0\right)-X\left(k_{x}=\pi / p_{x}, k_{z}=0\right)-K\left(k_{x}=\right.$ $\left.\pi / p_{x}, k_{z}=\pi / p_{z}\right)-M\left(k_{x}=0, k_{z}=\pi / p_{z}\right)-\Gamma\left(k_{x}=0, k_{z}=0\right)$.

propagation vectors for obtaining the band diagram (Fig. 2) and isofrequency dispersion relations (Fig. 3).

In the band diagram (Fig. 2) the first three modes are marked and the isofrequency dispersion curves for the three modes are presented in Fig. 3. The first mode starts from the $\Gamma$ point $\left(k_{x}=0, k_{z}=0\right)$ and has isofrequency curves resembling hyperboloids, as shown in Fig. 3(a). The second mode starts from $13.6 \mathrm{GHz}$, which also denotes the plasma frequency $\omega_{p}$. In our previous work [26] only the second mode was considered and the first mode was not excited.

From the hyperbolic isofrequency surfaces of mode 1 [Fig. 3(a)] we can confirm that the material acts as a hyperbolic metamaterial. However, at low frequencies the topology is more cylindrical than hyperbolic. The Poynting vector, which gives the direction of power flow, is normal to these isofrequency surfaces, so at low frequencies most power is confined in the $x-y$ plane. It is consistent with the fact that at low frequencies the structure behaves like a parallel plate waveguide and most of the power is confined between two grids. Mode 2 [Fig. 3(b)] and mode 3 [Fig. 3(c)] start as spheres of small radius at the plasma frequency $\left(\omega_{p}\right)$ around 13.6 GHz. The radius of these spheres can be expressed as $k_{0} n$, where $n$ is the effective refractive index and $k_{0}=\omega / c$ is the wave number in free space. A small radius implies a small effective refractive index $n$. Thus at frequencies slightly greater than the plasma frequency $13.6 \mathrm{GHz}$, where the second and third mode in the band diagram (Fig. 2) originate, the material acts as an epsilon-near-zero or index-near-zero medium [26].

\section{DENSITY-OF-STATES CALCULATION FROM ISOFREQUENCY SURFACES}

Having obtained the isofrequency surfaces, we now proceed to calculate the density of states for our metamaterial unit cell from these isofrequency surfaces. The density of states is defined as

$$
D(\omega)=\sum_{n} \int_{\mathrm{BZ}} d \mathbf{k} \delta\left(\omega-\omega_{n, \mathbf{k}}\right) .
$$

Integration is performed over $\mathbf{k}$ where $\omega_{n, \mathbf{k}}$ are the eigenfrequencies corresponding to the wave vectors $\mathbf{k}$ and mode number $n$. The DOS can also be expressed in terms of the infinitesimal volume of the shell formed between two closely separated isofrequency surfaces of frequencies $\omega$ and $\omega+d \omega$ :

$$
D(\omega) d \omega=\frac{1}{(2 \pi)^{3}} \iint_{\delta \omega} d \mathbf{k}_{\mathbf{1}} / / d \mathbf{k}_{\mathbf{2}} / / \frac{d \omega}{\left|\vec{\nabla} \omega_{k}\right|},
$$

where $\mathbf{k}_{\mathbf{1}} / /$ and $\mathbf{k}_{\mathbf{2}} / /$ are tangential to the isofrequency surface and $d \mathbf{k}_{\mathbf{1}} / / d \mathbf{k}_{\mathbf{2}}{ } / /$ forms a differential surface area element on the isofrequency surface $\omega\left(k_{x}, k_{y}, k_{z}\right)$. The integral in Eq. (15) can be evaluated by discretizing the isofrequency surface (Fig. 4) into $N$ triangles and expressing Eq. (15) as a summation:

$$
D(\omega)=\frac{1}{(2 \pi)^{3}} \sum_{i}^{N} \Delta^{i} \frac{1}{\left|\vec{\nabla} \omega_{k}^{i}\right|},
$$

where $\Delta^{i}$ is the area of the $i$ th triangular patch and $\left|\vec{\nabla} \omega_{k}^{i}\right|$ is the magnitude of gradient at the center of the triangle.

The density of states $D(\omega)$ calculated from the isofrequency surfaces shown in Fig. 3 using Eq. (16) is shown in Fig. 5. The density of states of individual modes of the metamaterial unit cell is compared with the density of states for vacuum for TM polarization given by $\frac{\omega^{2}}{2 \pi^{2} c^{3}}$. We observe that the DOS for mode 1 is large compared to vacuum which is expected due to the hyperbolic nature of the mode. In contrast, for modes 2 and $3 D(\omega)=0$ below $\omega_{p} \approx 13.6 \mathrm{GHz}$ as $\mathrm{TE}_{z}$ modes cannot propagate at these frequencies.
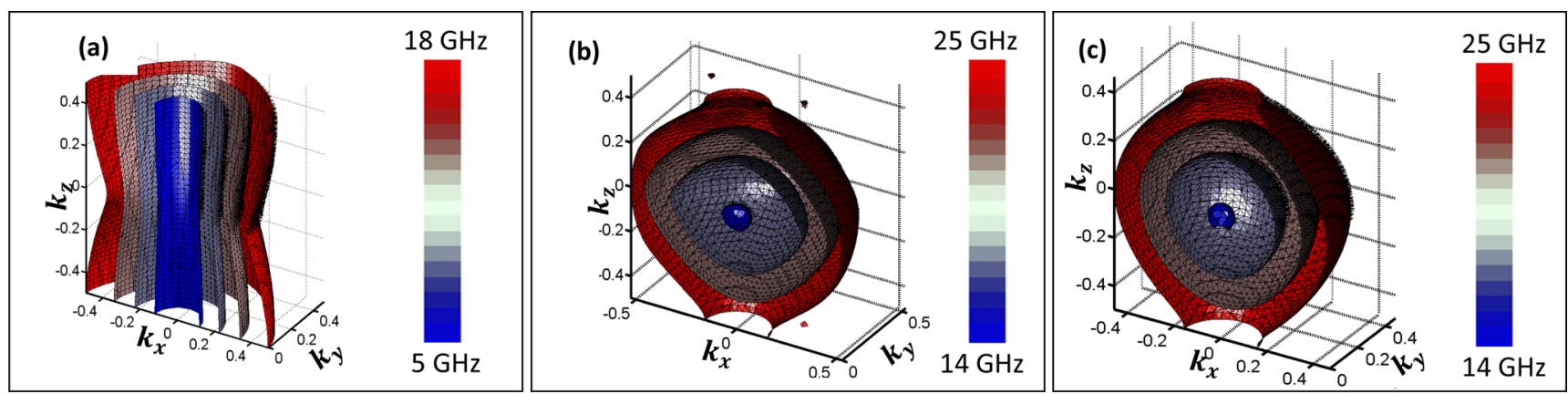

FIG. 3. Isofrequency surfaces for the unit cell in Fig. 1. Here, (a), (b), and (c) show isofrequency surfaces for modes 1, 2, and 3, respectively, as marked in Fig. 2. The topology of isofrequency surfaces for mode 1 (a) is hyperbolic and leads to a high density of states. Mode 2 (b) and mode 3 (c) begin at $13.6 \mathrm{GHz}$, at the plasma frequency $\omega_{p}$. The Brillouin zone sectioned by the plane $k_{y}=0$. 


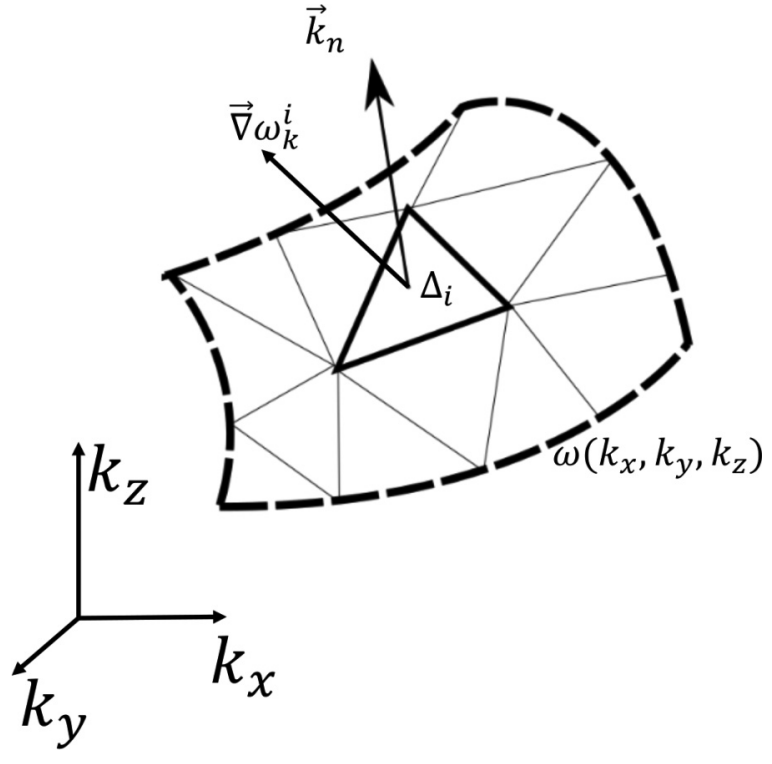

FIG. 4. Illustration of the method used to compute the density of states. The isofrequency surface, $\omega\left(k_{x}, k_{y}, k_{z}\right)$ is divided into $N$ triangles; the gradient $\vec{\nabla} \omega_{k}$ is computed at the centroids of the triangular patches and used in Eq. (16) to compute the density of states.

\section{EXPERIMENTS: MEASUREMENT OF THE PURCELL FACTOR WITH ANTENNA IMPEDANCE}

We now outline our procedure for the measurement of the Purcell factor from $S$-parameters. The sketch of the setup used for measurements is shown in Fig. 6. The dipole attached to a coaxial cable can be modeled as a one-port network system. The inset in Fig. 6 shows the experimental system as a one-port network. The dipole of impedance $Z$ is driven by a voltage source $V_{s}$, of reference impedance $Z_{w}$. Our experimental

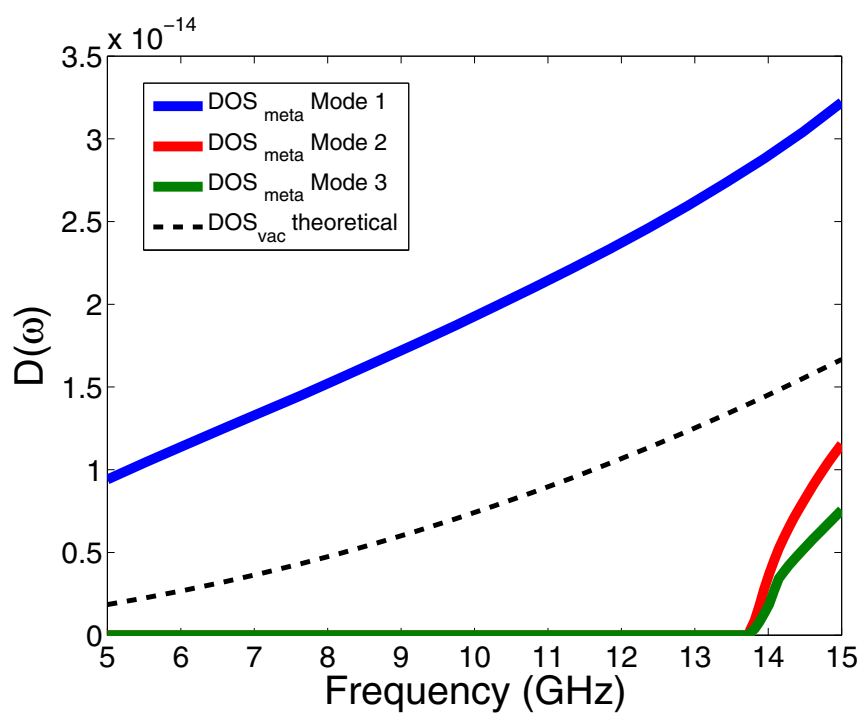

FIG. 5. Density of states computed with the isofrequency surfaces for mode 1 (blue), mode 2 (red), and mode 3 (green) as shown in Fig. 3 and compared with the theoretical density of states for vacuum (dashed). The first mode due to hyperbolic isofrequency surfaces has higher DOS than vacuum.
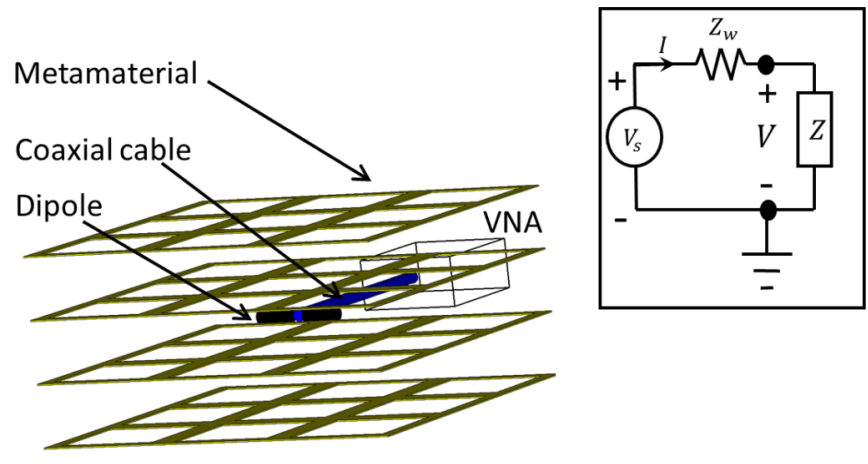

FIG. 6. Sketch of the experimental setup demonstrating the measurement of the Purcell factor with the dipole. Inset: The one-port network with the reference impedance $Z_{w}$.

system was calibrated to have a reference impedance equal to $50 \Omega$. The current flowing in the circuit is denoted by $I$. If the current flowing in the circuit $I$ is expressed as a superposition of incident current $I_{i}$ and reflected current $I_{r}$, $I=I_{i}-I_{r}$. For a one-port network system $S_{11}$ denotes the reflection coefficient. The reflection coefficient $S_{11}$ relates the incident and the reflected currents as $I_{i}=S_{11} I_{r} . S_{11}$ is measured from a vectorial network analyser (VNA) and is related to the impedances $Z$ and $Z_{w}$ as [43]

$$
S_{11}=\frac{Z-Z_{w}}{Z+Z_{w}} .
$$

The impedance of a dipole antenna for the purpose of evaluating the Purcell factor is obtained by measuring the scattering coefficient $S_{11}$. For a weakly lossy dipole antenna the real component of impedance $\operatorname{Re}(Z)$ can be approximated by the input impedance $R_{\text {in }}$ [24]. The Purcell factor using Eq. (11) can thus be measured as

$$
F=\frac{\operatorname{Re}(Z)}{\operatorname{Re}(Z)_{0}}=\frac{R^{\text {in }}}{R_{0}^{\text {in }}},
$$

where

$$
R^{\text {in }}=Z_{w} \frac{1-\operatorname{Re}\left(S_{11}\right)^{2}-\operatorname{Im}\left(S_{11}\right)^{2}}{\left[1-\operatorname{Re}\left(S_{11}\right)\right]^{2}+\operatorname{Im}\left(S_{11}\right)^{2}} .
$$

Here $S_{11}$ is the reflection coefficient and $Z_{w}=50 \Omega$ is the characteristic impedance. We use this approach to measure the Purcell factor of our metamaterial structure.

The procedure for measurement of the Purcell factor is as follows. The dipole (electric or magnetic) is attached to a coaxial cable and $S_{11}$ is recorded in free space. From $S_{11}$ measurements, the reference input resistance for vacuum $R_{\mathrm{in}, 0}$ is obtained via Eq. (19). The dipole is then placed at the center of the structure between the second and third copper grids. $S_{11}$ is recorded and $R_{\text {in }}$ is calculated again from Eq. (19). The Purcell factor is then obtained from Eq. (18) as the ratio of radiation resistance $R_{\text {in }}$ in the metamaterial to the $R_{\text {in }, 0}$ in vacuum. The electric and magnetic Purcell factors $\left(F^{e / m}\right)$ thus obtained are compared against the Purcell factor obtained using simulated $S_{11}$ from FDTD method (Fig. 10).

In simulations and for the experiments, the subwavelength size electric dipole antenna was made of thin conducting wires of total length $6.3 \mathrm{~mm}$, while the magnetic dipole was a wire loop antenna of radius $3.0 \mathrm{~mm}$. Measurements were performed 
with a VNA model Anritsu MS2027C for the frequency range 5-15 GHz. At the high-frequency end this was limited by the VNA. The plasma frequency $\left(\omega_{p}=13.6 \mathrm{GHz}\right)$ of the structure lies in this frequency range which allows us to observe the change in Purcell factor around this frequency.

Numerical simulations for calculations of the Purcell factor using the antenna impedance method were performed using the finite difference time domain solver available in CST. Perfectly matched layers are used to truncate the computational domain with space added near the structure. The total number of mesh cells was approximately $10^{9}$ for the entire structure. We checked numerically that the dipole is sufficiently subwavelength in length and the Purcell factor does not depend significantly on the dipole parameters. The available time domain solver in CST was used to calculate the scattering coefficients ( $S$-parameters) in the frequency range 5-15 GHz.

When the electric dipole is oriented along $\hat{z}$, the Purcell factor $(F)$ is strongly affected by the Fabry-Perot (FP) resonances. The FP resonances occur due to the finite lateral size of the structure. The effect of FP resonances on the Purcell factor in metamaterials has been studied in the context of wiremedium hyperbolic metamaterials [21] where it was argued that the Fabry-Perot resonances do not affect the average value of the Purcell factor. It was numerically shown that the frequency averaged Purcell factor in finite structure was in agreement with the Purcell factor of an infinitely periodic structure. While taking the average may seem heuristic, a similar signal processing technique can be justified from the physics of the resonances: The Fabry-Perot resonances are caused by reflections from the boundaries of the finite structure; eliminating these reflections by zeroing out the time signal from the time any reflections arrive should give a good approximation to the response of the infinite medium. A similar technique was used to remove Fabry-Perot resonance artifacts in hyperlens images [44].

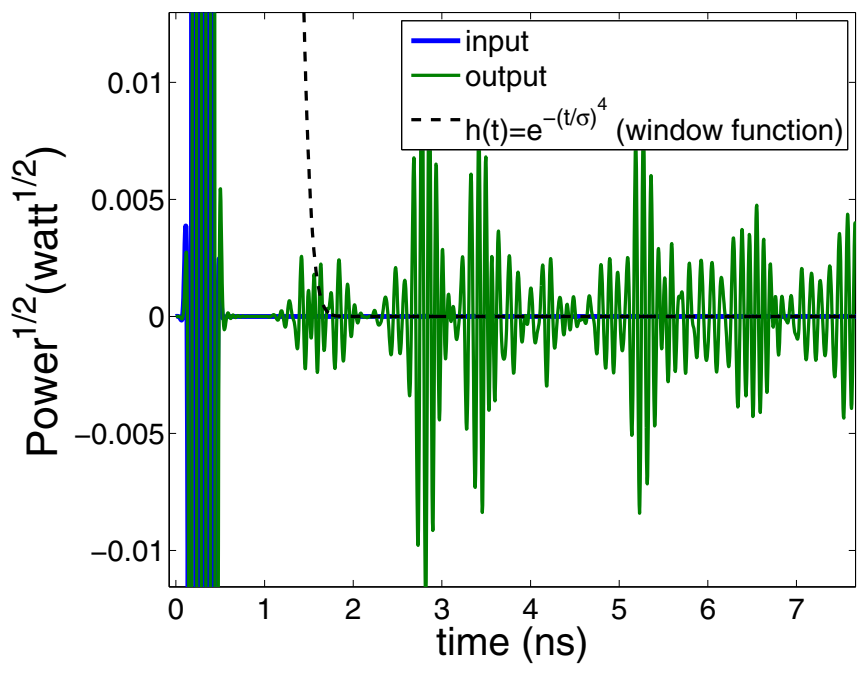

FIG. 7. Input and output time signals from FDTD simulations for an electric dipole along $\hat{z}$ placed at the center of the structure. The first reflection in the output signal occurs around $0.9 \mathrm{~ns}$, which corresponds to the time taken by the wave to reflect back from the boundary of the structure.

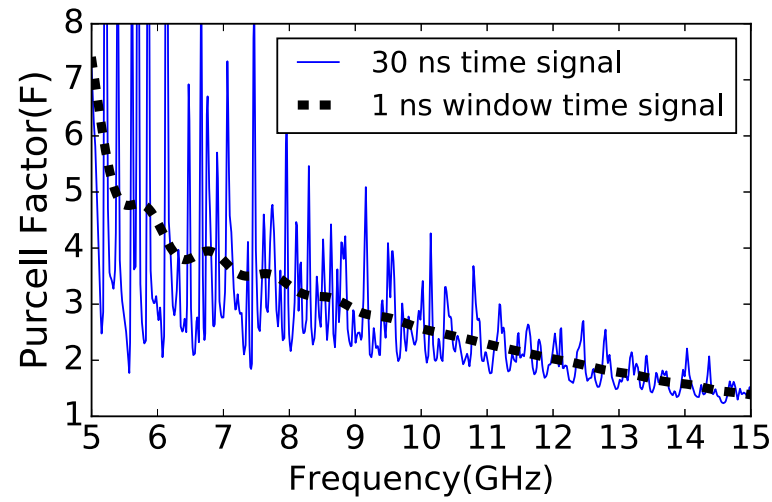

FIG. 8. The Purcell factor for an electric dipole along $\hat{z}$ computed from the time signals in Fig. 7. Solid curves are calculated from time signals up to $30 \mathrm{~ns}$; dashed curve gives the corresponding results after time windowing to eliminate the effect of the reflections from the boundaries.

The Purcell factor is calculated from the reflection coefficient $S_{11}$ [Eq. (19)]. In the FDTD simulations $S_{11}$ is calculated from the time signals as the ratio of the Fourier transform $(\mathfrak{F})$ of the output $[O(t)]$ and the input $[I(t)]$ time signals, $S_{11}(\omega)=\mathfrak{F}[O(t)] / \mathfrak{F}[I(t)]$. The dipole is placed at the center of the structure which has a lateral width of $L=280 \mathrm{~mm}$. The time for the wave to reflect back from the boundaries $\left(t^{\text {ref }}\right)$ is thus $t^{\text {ref }}=L / c \simeq 0.9 \mathrm{~ns}$ where $c$ is the speed of light in free space. We can observe the reflections in the time signals at $0.9 \mathrm{~ns}$ in Fig. 7. By limiting our time signal to $t^{\text {ref }}$ the reflections are excluded and Fabry-Perot resonances are eliminated. In order to limit the time signals to $t^{\text {ref }}$ we multiply the input and output signals by a super-Gaussian windowing function $h(t)=e^{-(t / \sigma)^{4}}$ with window length $\sigma=0.9 \mathrm{~ns}$. Since we obtain $S_{11}$ in the frequency domain from the VNA, we convolve the measured $S_{11}$ in frequency domain by $H(\omega)$ where $H(\omega)=\mathfrak{F}[h(t)]$. Figure 8 shows that the truncation of the time signal (Fig. 7) removes the effect of the Fabry-Perot resonances on the Purcell factor $(F)$.

\section{RESULTS AND DISCUSSION}

\section{A. Purcell factor for the magnetic dipole oriented along $\hat{z}$}

Figure 10(a) shows the measured magnetic Purcell factor for a magnetic dipole oriented along $\hat{z}$ inside the structure. A magnetic dipole embedded inside a uniaxial anisotropic medium oriented parallel to the symmetry axis $(\hat{z})$ emits in the $\mathrm{TE}_{\mathrm{z}}$ polarization [41] which allows no propagating solutions when $\omega<\omega_{p} \approx 13.6 \mathrm{GHz}$ where $\varepsilon_{/ /}<0$ [Eq. (12)]. Indeed, we see that below the plasma frequency the Purcell factor is close to zero because emission is suppressed, and only takes significant values above the plasma frequency. This result is analogous to the inhibition of spontaneous emission of atoms [5].

The enhancement in Fig. 10(a) around $8.5 \mathrm{GHz}$ is due to the antiresonance of the dipole which occurs when the circumference of the dipole loop $C=\lambda / 2$. This is illustrated in Fig. 9, which shows the impedance of a magnetic dipole loop antenna with outer radius $b=3 \mathrm{~mm}$ and wire radius $a=2 \mathrm{~mm}$, using a Fourier series expansion method $[45,46]$ 


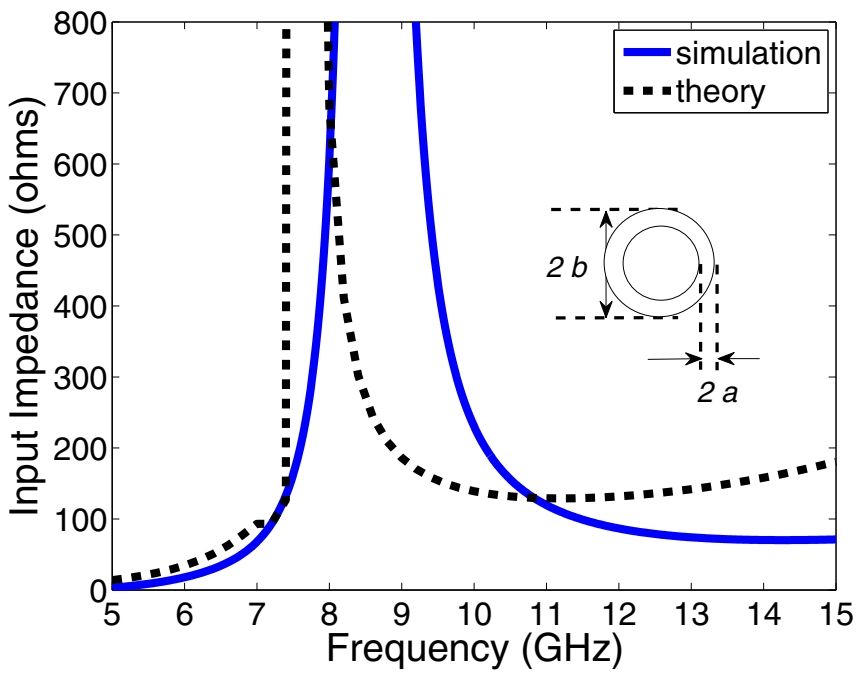

FIG. 9. Comparison of FDTD simulations (blue) and theoretical calculations (green) of input impedance $\operatorname{Re}(Z)$ of a magnetic dipole antenna in vacuum with outer radius $b=3 \mathrm{~mm}$ and wire radius $a=1 \mathrm{~mm}$.

(green curve) and an FDTD calculation (blue curve). These confirm that the peak around $8.5 \mathrm{GHz}$ corresponds to the first antiresonance.

The Purcell factor is a property of the medium and cannot depend on the dipole. Hence the peak near $8.5 \mathrm{GHz}$ in Fig. 10(a) does not correspond to an enhancement of the Purcell factor. This is analogous to the strong-coupling regime where the LDOS loses its meaning. Hence, care must be taken to avoid antenna-related artifacts when using the impedance method to measure the Purcell factor.

\section{B. Purcell factor for the electric dipole oriented along $\hat{y}$}

In Fig. 10(b) the measurement of the Purcell factor for an electric dipole perpendicular to the anisotropy axis along $\hat{y}$ is shown. In this particular orientation the field is a superposition of $\mathrm{TE}_{\mathrm{z}}$ and $\mathrm{TM}_{\mathrm{z}}$ polarizations. The Purcell factor is similar to that of the magnetic dipole parallel to the anisotropy axis (pure $\mathrm{TE}_{\mathrm{z}}$ polarization) [Fig. 10(a)], without the antiresonance peak. The similarity with the magnetic Purcell factor can be interpreted as follows. For a magnetic dipole oriented along $\hat{z}$, the electric field is in the $x-y$ plane $\left(\mathrm{TE}_{\mathrm{z}}\right.$ polarization). For the electric dipole along $\hat{y}$, perpendicular to the anisotropy axis $\hat{z}$, most of the power is radiated with the electric field along $\hat{y}$. However, the structure in which it is embedded does not allow propagation with a component of the electric dipole along $\hat{y}$, below the plasma frequency as the metallic grids are in the $x-y$ plane similar to the magnetic dipole. Hence we measure the same plasma frequency in both cases and also a similar Purcell factor with the electric dipole perpendicular to the anisotropy axis.

We point out that there is no enhancement of the Purcell factor around $8.5 \mathrm{GHz}$ for this case, in contrast to what was observed for the magnetic dipole [Fig. 10(a)]. Indeed, for an electric dipole of length $l=6 \mathrm{~mm}$, the antiresonance lies at the matching frequency $\lambda / 2=l$ (that is, $25 \mathrm{GHz}$ ) which is outside the measurement range.
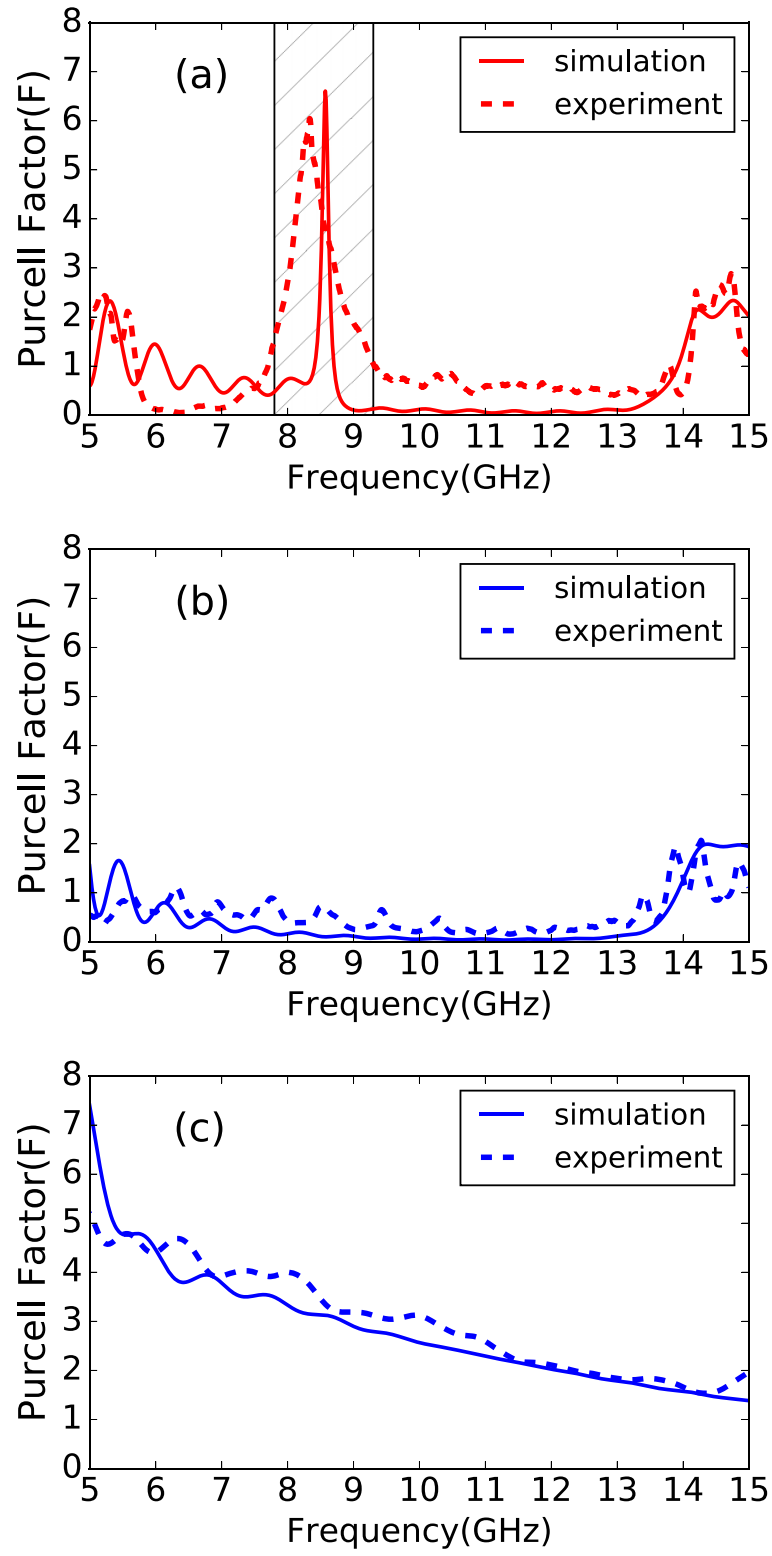

FIG. 10. Comparison of measurements (dashed) and FDTD simulations (solid) of Purcell factors obtained using the impedance method for different dipole orientations placed inside the hyperbolic metamaterial. (a) Purcell factor for a magnetic dipole parallel to the anisotropy axis $z$ ( $\mathrm{TE}_{\mathrm{z}}$ polarization). The hatching represents the frequency of the anti-resonance of the magnetic dipole. (b) Purcell factor for an electric dipole along $\hat{y}$ ( $\mathrm{TE}_{\mathrm{z}}$ polarization). (c) Purcell factor for an electric dipole parallel to the anisotropy axis $\hat{z}\left(\mathrm{TM}_{\mathrm{z}}\right.$ polarization).

\section{Purcell factor for the electric dipole oriented along $\hat{z}$}

The Purcell factor measured with the electric dipole oriented along $\hat{z}$ in the structure is shown in Fig. 10(c). The electric dipole embedded inside a uniaxial anisotropic medium oriented parallel to the anisotropy axis emits in the $\mathrm{TM}_{\mathrm{z}}$ polarization [41]. The Fabry-Perot resonances are removed by applying a convolution which corresponds to a super-Gaussian window in the time domain, with a window length of $t^{\mathrm{ref}}=0.9 \mathrm{~ns}$ as mentioned in Sec. VI. In the $\mathrm{TM}_{\mathrm{z}}$ polarization the dispersion relation for the uniaxial hyperbolic 
medium is given by Eq. (13). The hyperbolic nature of the dispersion relation leads to a large LDOS and a large Purcell factor. The Purcell factor decreases with frequency, which can be interpreted as a consequence of the periodicity of the structure limiting the maximum permitted value of $k_{z}$. In a medium where $k_{z}$ is bound by a maximum value $k_{z, \max }$ the DOS of the structure is linearly proportional to $\omega$ [47]:

$$
D(\omega)=\frac{\omega}{\pi^{2} c^{2}} \frac{\varepsilon_{z z}}{2} k_{z, \max },
$$

whereas the DOS for vacuum is proportional to $\omega^{2}$. The Purcell factor is the ratio of LDOS inside the structure to the LDOS in vacuum, and hence decreases with frequency $\omega$. The enhancement of the Purcell factor is not extremely large as would be expected for a truly hyperbolic mode, but we note that the structure was not designed with the specific aim of enhancing the Purcell factor.

\section{Comparison of the DOS from eigenmode calculations and impedance of antennas}

So far, we have calculated the DOS using eigenmode analysis (Sec. V), and have measured and determined the LDOS using the impedance method (Sec. VI). The LDOS can be obtained from eigenmode calculations, as discussed in Ref. [48] Conversely, the DOS can be calculated from the LDOS quite readily, allowing us to compare both results. We define the relative density of states (rel. DOS) as the ratio of the DOS of the metamaterial unit cell to the theoretical DOS for vacuum. The DOS is the spatial and orientational average of the LDOS over the unit cell. The Purcell factor depends on the location and the orientation of the dipole inside the unit cell. Hence, an average of the Purcell factor over the unit cell and the three orientations $(x, y$, and $z)$ is equivalent to rel. DOS.

For obtaining the rel. DOS with the impedance method, an average of the Purcell factor over a unit cell of the metamaterial is required. Numerical simulations of a dipole antenna were performed at 27 locations in one-eighth of the unit cell along $\hat{z}$ and $\hat{y}$ directions. By symmetry of the unit cell it is equivalent to $27 \times 8=216$ locations. The impedance was obtained from reflection coefficient $S_{11}$ and the average of the Purcell factor was calculated. The length of the dipole antenna was chosen to be $1 \mathrm{~mm}$. The Purcell factor is obtained from the impedance method (Sec. VI) at each location and its average is denoted as $\left\langle F_{i}\right\rangle$, where $i$ is the orientation of the dipole. The simulation was limited to $t^{\text {ref }}=0.9 \mathrm{~ns}$ to remove reflections from the boundaries, as discussed in Sec. VI.

Averaging the Purcell factor in the three directions we obtain rel. DOS $=\left(\left\langle F_{x}\right\rangle+\left\langle F_{y}\right\rangle+\left\langle F_{z}\right\rangle\right) / 3=\left(2\left\langle F_{/ /}\right\rangle+\right.$ $\left.\left\langle F_{z}\right\rangle\right) / 3$. For the eigenmode method, there are three modes present between 5 and $15 \mathrm{GHz}$ (Fig. 5) and they are summed to obtain rel. DOS, that is, rel. DOS $=\left(\mathrm{DOS}^{\mathrm{Meta}}\right) / \mathrm{DOS}^{\mathrm{vac}}=$ $\left(\mathrm{DOS}^{\text {Mode } 1}+\mathrm{DOS}^{\text {Mode2 }}+\mathrm{DOS}^{\text {Mode 3}}\right) / \mathrm{DOS}^{\mathrm{vac}}$. Results of the comparison are presented in Fig. 11 and we find a good agreement between the two methods.

\section{CONCLUSION}

A detailed analysis of the Purcell factor inside a fishnet metamaterial at microwave frequencies has been presented.

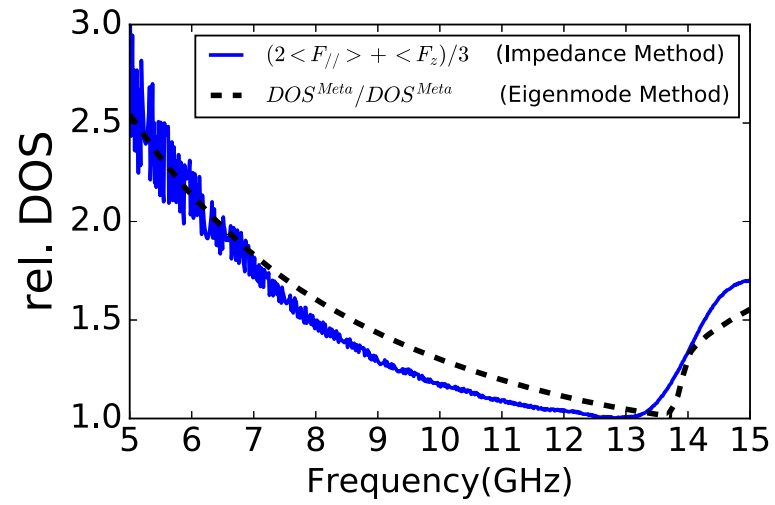

FIG. 11. Comparison of the rel. DOS obtained from impedance method (blue) and rel. DOS from eigenmode calculations (dashed) as shown in Fig. 5.

Measurements and numerical calculations of impedance of antennas located inside the metamaterial were used to determine the Purcell factor. The anisotropic nature of the structure leads to different electric and magnetic Purcell factors due to distinct dispersion relations in the $\mathrm{TM}_{z}$ and $\mathrm{TE}_{z}$ polarizations. We performed eigenmode calculations of the periodic unit cell of the metamaterial structure to obtain the band structure and the isofrequency surfaces. From the isofrequency surfaces, we confirmed the presence of a hyperboliclike mode, which causes an enhancement of the Purcell factor, and outlined a method to calculate the density of states of the metamaterial unit cell from the isofrequency surfaces.

We show that the impedance method can be used to obtain the density of states of the metamaterial. We numerically calculated the DOS by averaging the Purcell factor obtained from impedance calculations over the unit cell of the metamaterial. We compared the DOS obtained from the impedance method with the DOS obtained from eigenmode calculations of the unit cell. By limiting the simulation time the artifacts due to reflections are removed. It eliminates the FP resonances, and the rel. DOS for a finite structure and that for an infinite structure agree with each other. Though we studied the density of states of a fishnet metamaterial at microwave frequencies, our method can be used to study the density of states for other periodic structures like a wire medium as well. The impedance measurements of an antenna can be developed as a useful tool to study the density of states of any medium.

\section{ACKNOWLEDGMENTS}

Research was conducted within the context of the International Associated Laboratory "ALPhFA: Associated Laboratory for Photonics between France and Australia." This work was carried out thanks to the support of the A*MIDEX project (Grant No. ANR-11-IDEX-0001-02) funded by the Investissements d'Avenir French Government program, managed by the French National Research Agency (ANR). This work was supported by the Australian Research Council (CUDOS Centre of Excellence CE110001018). The authors thank Elodie Georget and Pierre Sabouroux for help with microwave measurements and John Sipe for valuable discussions. 
[1] E. Purcell, Phys. Rev. 69, 681 (1946).

[2] K. H. Drexhage, J. Lumin. 1-2, 693 (1970).

[3] R. R. Chance, A. Prock, and R. Silbey, Adv. Chem. Phys. 37, 65 (1978).

[4] D. Kleppner, Phys. Rev. Lett. 47, 233 (1981).

[5] R. G. Hulet, E. S. Hilfer, and D. Kleppner, Phys. Rev. Lett. 55, 2137 (1985)

[6] E. Yablonovitch, Phys. Rev. Lett. 58, 2059 (1987).

[7] M. D. Leistikow, A. P. Mosk, E. Yeganegi, S. R. Huisman, A. Lagendijk, and W. L. Vos, Phys. Rev. Lett. 107, 193903 (2011).

[8] H. P. Schriemer, H. M. van Driel, A. Femius Koenderink, and W. L. Vos, Phys. Rev. A 63, 011801 (2000).

[9] J. Wijnhoven and W. L. Vos, Science 281, 802 (1998).

[10] V. S. C. Manga Rao and S. Hughes, Phys. Rev. B 75, 205437 (2007).

[11] J. T. Choy, B. J. M. Hausmann, T. M. Babinec, I. Bulu, M. Khan, P. Maletinsky, A. Yacoby, and M. Lonar, Nat. Photon 5, 738 (2011).

[12] I. S. Maksymov, M. Besbes, J. P. Hugonin, J. Yang, A. Beveratos, I. Sagnes, I. Robert-Philip, and P. Lalanne, Phys. Rev. Lett. 105, 180502 (2010).

[13] M. Khajavikhan, A. Simic, M. Katz, J. H. Lee, B. Slutsky, A. Mizrahi, V. Lomakin, and Y. Fainman, Nature (London) 482, 204 (2012).

[14] S. Kuhn, U. Hakanson, L. Rogobete, and V. Sandoghdar, Phys. Rev. Lett. 97, 017402 (2006).

[15] J. Azoulay, A. Débarre, A. Richard, and P. Tchénio, Europhys. Lett. 51, 374 (2000).

[16] C. Sauvan, J. P. Hugonin, I. S. Maksymov, and P. Lalanne, Phys. Rev. Lett. 110, 237401 (2013).

[17] X. Zambrana-Puyalto and N. Bonod, Phys. Rev. B 91, 195422 (2015).

[18] J. M. Gérard, B. Sermage, B. Gayral, B. Legrand, E. Costard, and V. Thierry-Mieg, Phys. Rev. Lett. 81, 1110 (1998).

[19] Z. Jacob, I. I. Smolyaninov, and E. E. Narimanov, Appl. Phys. Lett. 100, 181105 (2012).

[20] A. Poddubny, I. Iorsh, P. Belov, and Y. Kivshar, Nat. Photon 7, 948 (2013).

[21] S. Kosulnikov, D. Filonov, S. Glybovski, P. Belov, S. Tretyakov, and C. Simovski, IEEE Trans. Antennas Propag. 63, 4848 (2015).

[22] A. P. Slobozhanyuk, P. Ginzburg, D. A. Powell, I. Iorsh, A. S. Shalin, P. Segovia, A. V. Krasavin, G. A. Wurtz, V. A. Podolskiy, P. A. Belov, and A. V. Zayats, Phys. Rev. B 92, 195127 (2015).

[23] J.-J. Greffet, M. Laroche, and F. Marquier, Phys. Rev. Lett. 105, 117701 (2010).
[24] A. E. Krasnok, A. P. Slobozhanyuk, C. R. Simovski, S. A. Tretyakov, A. N. Poddubny, A. E. Miroshnichenko, Y. S. Kivshar, and P. A. Belov, Sci. Rep. 5, 12956 (2015).

[25] A. P. Slobozhanyuk, A. N. Poddubny, A. E. Krasnok, and P. A. Belov, Appl. Phys. Lett. 104, 161105 (2014).

[26] S. Enoch, G. Tayeb, P. Sabouroux, N. Guérin, and P. Vincent, Phys. Rev. Lett. 89, 213902 (2002).

[27] B. H. Bransden and C. J. Joachain, Physics of Atoms and Molecules (Addison-Wesley, New York, 1983).

[28] R. Carminati, A. Cazé, D. Cao, F. Peragut, V. Krachmalnicoff, R. Pierrat, and Y. De Wilde, Surf. Sci. Rep. 70, 1 (2015).

[29] J. D. Jackson, Classical Electrodynamics Third Edition (Wiley, New York, 1998).

[30] L. Novotny and B. Hecht, Principles of Nano-Optics (Cambridge University, Cambridge, England, 2012).

[31] W. Demtröder, Atoms, Molecules and Photons, Graduate Texts in Physics (Springer, Berlin, 2010).

[32] J. B. Pendry, A. J. Holden, D. J. Robbins, and W. J. Stewart, J. Phys.: Condens. Matter 10, 4785 (1998).

[33] G. Guida, D. Maystre, G. Tayeb, and P. Vincent, J. Opt. Soc. Am. B 15, 2308 (1998).

[34] D. Felbacq and G. Bouchitté, Waves Random Media 7, 245 (1997).

[35] D. R. Smith and D. Schurig, Phys. Rev. Lett. 90, 077405 (2003).

[36] A. M. Nicolson and G. F. Ross, IEEE Trans. Instrum. Meas. 19, 377 (1970).

[37] W. B. Weir, Proc. IEEE 62, 33 (1974).

[38] Z. Jacob, J.-Y. Kim, G. V. Naik, A. Boltasseva, E. E. Narimanov, and V. M. Shalaev, Appl. Phys. B 100, 215 (2010).

[39] P. Shekhar, J. Atkinson, and Z. Jacob, Nano Convergence 1, 1 (2014).

[40] C. L. Cortes, W. Newman, S. Molesky, and Z. Jacob, J. Opti. 14, 063001 (2012).

[41] P. C. Clemmow, Proc. Inst. Elect. Eng. 110, 101 (1963).

[42] CST Microwave Studio, http://www.cst.com, 2015.

[43] R. J. Collier and A. D. Skinner, Microwave Measurements (IET, London, 2007).

[44] K. J. Kaltenecker, A. Tuniz, S. C. Fleming, A. Argyros, B. T. Kuhlmey, M. Walther, and B. M. Fischer, Optica 3, 458 (2016).

[45] C. A. Balanis, Antenna Theory: Analysis and Design, 2nd ed. (Wiley, New York, 1997).

[46] J. E. Storer, AIEE Trans. 75, 606 (1956).

[47] S.-A. Biehs, S. Lang, A. Y. Petrov, M. Eich, and P. BenAbdallah, Phys. Rev. Lett. 115, 174301 (2015).

[48] I. S. Nikolaev, W. L. Vos, and A. F. Koenderink, J. Opt. Soc. Am. B 26, 987 (2009). 